\title{
Natural Therapeutics Targeting Survivin
}

\section{Jagat R Kanwar*, Rasika M Samarasinghe and Rupinder K Kanwar}

Nanomedicine-Laboratory of Immunology \& Molecular Biomedical Research (LIMBR), Centre for Biotechnology and Interdisciplinary Biosciences, Institute for Frontier Materials (IFM), Deakin University, Geelong, Australia

Ardisianone, a naturally derived compound from the roots and stems of Ardisia virens Kurz (Myrsinaceae) was studied for its anticancer effects on human hormone-refractory prostate cancer (HRPC) cells and other prostate cancer cells lines (PC-3 and DU145). It was shown that Arsisianone dose dependently inhibited cell growth and induced apoptosis of cells. It was also able to down regulate survivin and activate mitochondrial associated caspases [1]. Recently, vitamin $\mathrm{E}$ was shown to inhibit the up regulation of survivin expression in pancreactic cells and induced apoptosis. Vitamin E treatment significantly down regulated the expression of XIAP (X-linked inhibitor of apoptosis protein) levels with an even higher inhibition of survivin expression. Cells transfected with $100 \mathrm{nM}$ survivin siRNA and treated with vitamin $\mathrm{E}(80 \mu \mathrm{M})$ showed reduced expression of survivin as compared to the control treatments [2]. Another study used quercetin, a plant derived flavonoid, and tested its activity against nonHodgkin's lymphoma B cells. The study showed quercetin induced cell death in resistant transformed B cells by restoring the TRAILinduced apoptosis pathway. Quercetin also inactivated the production of survivin and degraded myeloid leukemia cell differentiation protein Mcl-1 [3].

An interesting study analysing the effect of curcumin on hepatocellular carcinoma cells revealed the decreased efficacy of curcumin treatment on cells when survivin is suppressed. Using siRNA methods, survivin was suppressed in HCC cells and curcumin was tested for its anticancer effects. Interestingly, the study demonstrated that survivin knockdown cells developed resistance to curcumin treatment as opposed to non-transfected HCC cells. Survivin-siRNA transfected cells had a higher survival rate following curcumin treatment and lower apoptotic levels as compared to the scrambled-siRNA transfected HCC cells. Although it is not known why suppression of survivin stimulates curcumin resistance in cancer cells, it was discussed that it may be linked to the heat shock $70 \mathrm{kDA}$ protein 5 (HSPA5) mechanisms. Survivin has a direct correlation to HSPA5 expression which in turns affects the therapeutic efficacy of curcumin on cells. Therefore, suppressing survivin reduces levels of HSPA5 which can therefore reduce the effect of curcumin treatment leading to resistance of cells to curcumin [4].

\section{Other Recent Therapeutics Targeting Survivin}

The survivin modulatory activity of SAHA (suberoylanilide hydroxamic acid), a histone deacetylase, was examined on a colon cancer animal model. I.p. administration of SAHA to Balb/c nude mice inhibited the growth of colon tumours and was found to directly inhibit the expression of survivin, cyclin D1 and cell proliferative proteins like Ki67 [5].

A novel strategy involved the use of a screening process to acquire interacting proteins for survivin. Their study found a novel protein from the domain of the ferritin heavy chain 1 (FTH1) that had high affinity to survivin. The FTH1-derived survivin interaction domain transfected to tumour cells significantly down regulated the expression of survivin which inhibited the growth of tumour cells. Further the recombinant protein supressed the growth of tumour in nude mice, significantly inhibited cell density upon extended exposure in tumour cells but had no cytotoxic effects on non-cancerous cell lines (MCF$10 \mathrm{a}$ and NIH/3T3) [6]. Another study used microRNA-203 on laryngeal carcinoma and tested its direct effect on survivin expression. miRNA-203 suppressed the proliferation of cancer cells and colony formation and was shown to directly inhibit the gene expression of survivin [7].

A nanotherapy study developed with survivin miRNA plasmid loaded human serum abumin (HAS) nanoparticles and examined its efficacy on inhibiting survivin expression and increasing radiation responsiveness in SW480 colon cancer cells. The nanoparticle system was able to significantly reduce survivin expression by $50 \%$ and induce cellular toxicity when given in combination with ionising radiation [8]

\section{Future Prospective for Survivin Targeting}

Currently, YM155 is the lead compound that has shown anticancer effects through the inhibition of survivin. Although it's found to be well tolerated and safe, its efficacy was reported to be low. Therefore, new therapeutics can involve using the combination of YM155 together with other survivin targeted molecules like dominant negative survivin or survivin antisense genes. These can also be conjugated with chemotherapeutic drugs like doxorubicin and cisplatin that can inhibit tumour growth. We can also use other antagonist molecules that inhibit survivin linked pathways such as EGFR, STAT3, HSP90, p53 or XIAP. Further to improve protection of molecules, they can be encapsulated in biodegradable polymeric nanoparticles. These particles can be conjugated with cancer cell targeted peptides such as heparan sulphate (CGKRK), VEGF receptor (ATWLPPR), erbB2 (LTVSPWY) and VCAM-1 (VHSPNKK) [9]. To enhance the penetration of survivin targeted molecules into cells, it can be conjugated to penetrating peptide such as R9, HIV-1, TAT, S413PV or HATF3.

\section{References}

1. Yu CC, Wu PJ, Hsu JL, Ho YF, Hsu LC, et al. (2012) Ardisianone, a natural benzoquinone, efficiently induces apoptosis in human hormone-refractory prostate cancers through mitochondrial damage stress and survivin downregulation. Prostate.

2. Patacsil D, Osayi S, Tran AT, Saenz F, Yimer L, et al. (2012) Vitamin E succinate inhibits survivin and induces apoptosis in pancreatic cancer cells. Genes Nutr 7: 83-89.

3. Jacquemin G, Granci V, GallouetAS, Lalaoui N, Morlé A, et al. (2012) Quercetinmediated Mcl-1 and survivin downregulation restores TRAIL-induced apoptosis in non-Hodgkin's lymphoma B cells. Haematologica 97: 38-46.

*Corresponding author: Jagat $\mathrm{R}$ Kanwar, Nanomedicine-Laboratory of Immunology \& Molecular Biomedical Research (LIMBR), Centre for Biotechnology and Interdisciplinary Biosciences, Institute for Frontier Materials (IFM), Deakin University, Geelong, Victoria 3217, Australia, Tel: 00613 52271148; Fax: 00613 52272539; E-mail: jagat.kanwar@deakin.edu.au

Received September 20, 2012; Accepted September 20, 2012; Published September 23, 2012

Citation: Kanwar JR, Samarasingie RM, Kanwar RK (2012) Natural Therapeutics Targeting Survivin. Biochem Anal Biochem 1:e121. doi:10.4172/21611009.1000e121

Copyright: (c) 2012 Kanwar JR, et al. This is an open-access article distributed under the terms of the Creative Commons Attribution License, which permits unrestricted use, distribution, and reproduction in any medium, provided the original author and source are credited. 
Citation: Kanwar JR, Samarasingie RM, Kanwar RK (2012) Natural Therapeutics Targeting Survivin. Biochem Anal Biochem 1:e121. doi:10.4172/21611009.1000e121

4. Hung CS, Liu HH, Huang MT, Cheng CW, Kuo LJ, et al. (2012) Knockdown Survivin Expression Reduces the Efficacy of Curcumin Treatment in Hepatocellular Carcinoma Cells. Ann Surg Oncol1 9: 3547-3555

5. Jin JS, Tsao TY, Sun PC, Yu CP, Tzao C (2012) SAHA inhibits the growth of colon tumors by decreasing histone deacetylase and the expression of cyclin D1 and survivin. Pathol Oncol Res 18: 713-720.

6. Weiss A, Brill B, Borghouts C, Delis N, Mack L, et al. (2012) Survivin inhibition by an interacting recombinant peptide, derived from the human ferritin heavy chain, impedes tumor cell growth. J Cancer Res Clin Oncol 138: 1205-1220.
7. Bian K, Fan J, Zhang X, Yang XW, Zhu HY, et al. (2012) MicroRNA-203 leads to $\mathrm{G} 1$ phase cell cycle arrest in laryngeal carcinoma cells by directly targeting survivin. FEBS Lett 586: 804-809.

8. Gaca S, Reichert S, Rödel C, Rödel F, Kreuter J (2012) Survivin-miRNAloaded nanoparticles as auxiliary tools for radiation therapy: preparation, characterisation, drug release, cytotoxicity and therapeutic effect on colorectal cancer cells. J Microencapsul.

9. Vivès E, Schmidt J, Pèlegrin A (2008) Cell-penetrating and cell-targeting peptides in drug delivery. Biochim Biophys Acta 1786: 126-138. 